\title{
Cortical responses to visual motion in alert and anesthetized monkeys
}

To THE EDITOR-Cortical area MT of macaque monkeys contains directionselective neurons. Some respond only to the components of complex moving patterns, whereas others compute true pattern motion ${ }^{1,2}$. In Nature, Pack et al. ${ }^{3}$ reported that most MT neurons compute pattern motion in alert macaques but signal only component motion under anesthesia, but we have found the prevalence of component- and pattern-selective neurons to be unaffected by anesthesia ${ }^{1,2,4}$. Pack et al. conclude that motion integration circuits are impaired by anesthesia ${ }^{3}$. We believe their results can be explained by their choice of stimuli and anesthetic.

A neuron is classified as componentselective if its responses to 'plaid' patterns (Fig. 1c) are proportional to the sum of its responses to the plaids' components ${ }^{1}$ (Fig. 1a and b). However, the plaids used by Pack et al. ${ }^{3}$ (Fig. 1d) are not the sum of the components they used. Where the gratings' bars intersect, the luminance of an additive plaid is doubled, but their stimulus had uniform luminance. A third component (Fig. 1e) must be subtracted from the gratings to create their nonadditive plaid. Classification using these stimuli requires that the components of the plaid move in a different direction than the plaid itself, but this third component moves in the same direction ${ }^{5}$ (Fig. 1e). The third component would elicit patternlike responses from all MT cells, and thus component-selective cells tested with this non-additive plaid would masquerade as pattern-selective. It is therefore inappropriate to use non-additive plaids for this classification method, and the use of these patterns led Pack et al. erroneously to claim that most MT neurons in alert animals are pattern-selective.

Why might anesthesia change patternlike into component-like responses? The third component is low in contrast compared to the grating components. Neurons in MT are very sensitive ${ }^{6}$, so such a low-contrast stimulus component would normally be effective. However, Pack et al. used isoflurane anesthesia, which substantially reduces contrast sensitivity in cortical and thalamic neurons while only modestly reducing responses to highcontrast stimuli ${ }^{7,8}$. Isoflurane also poten-
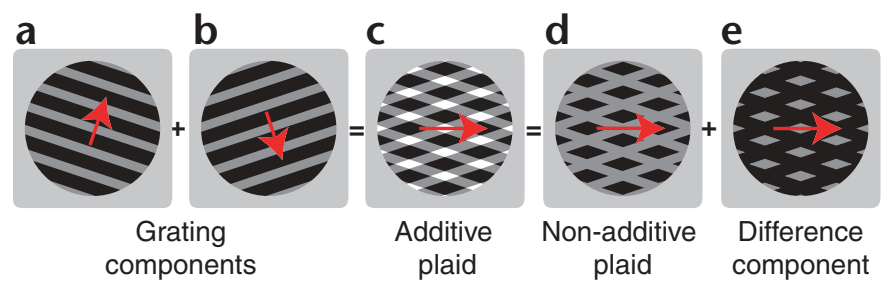

Fig. I. Additive and non-additive plaids. (a, b) Grating components of (c), an additive plaid. (d) The non-additive plaid used by Pack et al. ${ }^{3}$. (e) The component subtracted from (c) to create (d).

tiates GABA-mediated inhibition ${ }^{9}$, and thus strengthens the cross-orientation suppression characteristic of cortical responses ${ }^{10}$. These factors would selectively weaken responses to the third component (Fig. 1e). Thus, isoflurane would effectively convert non-additive plaids into additive plaids by selectively attenuating the third component. This is relevant only because Pack et al. used non-additive plaids; classification using standard additive plaids ${ }^{1}$ is unaffected by anesthesia.

We therefore suggest that the findings of Pack et al. ${ }^{3}$ do not reflect any fundamental property of cortical motion detection, but result instead from unfortunate choices of stimulus and anesthetic. The stimulus led Pack et al. to misclassify their neurons in alert animals, and the anesthetic caused this classification to change. These factors explain the apparent difference between their results ${ }^{3}$ and ours ${ }^{1,2,4}$.

\section{J. Anthony Movshon 1,2,}

Thomas D. Albright 1,3,

Gene R. Stoner ${ }^{3}$, Najib J. Majaj ${ }^{2}$ and

Matthew A. Smith ${ }^{2}$

${ }^{1}$ Howard Hughes Medical Institute

${ }^{2}$ Center for Neural Science, New York

University, New York, New York 10003, USA

${ }^{3}$ Systems Neurobiology Laboratories, The

Salk Institute for Biological Studies, La Jolla,

California 92037, USA

e-mail:movshon@nyu.edu

REPLY-Movshon et al. suggest that we found more pattern cells in alert monkeys because a "third component" in our stimuli "would elicit pattern-like responses from all MT cells." Although this explanation sounds plausible, it has been tested directly and found to be wrong. Stoner and Albright ${ }^{4}$ compared, for single MT neurons in alert monkeys, the responses to plaid stimuli with and without the third component. They found that introducing the third component caused MT responses to become less pattern-like and more component-like (Fig. 3b in ref. 4). Moreover, Stoner and Albright's third component was greater in amplitude than ours, so the explanation of Movshon et al. cannot be right.

The processing of plaid stimuli is particularly sensitive to small stimulus manipulations ${ }^{4,5,11}$, and MT neurons are sensitive to a wide variety of visual cues $^{12,13}$. Consequently, there is no reason to assume that the luminance of the grating intersections accounts for the different results in the different laboratories. The stimuli were not equated for background luminance, retinal eccentricity, stimulus size, grating angle, duty cycle, spatial frequency or contrast, and each of these parameters can strongly influence the processing of plaids ${ }^{5,11}$. At present, we simply do not know which of the stimulus differences is responsible for the different results, and neither do Movshon et al. It is precisely for this reason that we held all of these parameters constant in our comparison of the alert and anesthetized states.

The claims about isoflurane and contrast sensitivity from Movshon et al. are misleading. In both of the studies cited, the differential effects of isoflurane were confined to temporal frequencies $(1 \mathrm{~Hz}$ or less) much lower than those used in our study. For the range of frequencies we used $(5-10 \mathrm{~Hz})$, the contrast sensitivity found with isoflurane was similar to that 